\title{
Le continent oublié. Lumières et zones d'ombre des recherches sur la dissémination des plastiques
}

\author{
Denis Blot ${ }^{1}$ (D), Romain Tramoy ${ }^{2}$ (D), Johnny Gasperi ${ }^{3}$ (D) et Bruno Tassin ${ }^{4,}$ (B) \\ ${ }^{1}$ Sociologie, Université de Picardie Jules-Verne, UR Habiter le monde, Amiens, France \\ 2 Sciences de la Terre, Université Paris-Est Créteil, UR LEESU, Créteil, France \\ ${ }^{3}$ Chimie, Université Gustave-Eiffel, UR LEE, Nantes, France \\ ${ }^{4}$ Sciences de l'environnement, École des Ponts ParisTech, UR LEESU, Champs-sur-Marne, France
}

\begin{abstract}
Résumé - Pourquoi le nombre de publications scientifiques sur les plastiques a-t-il explosé sur les 7-8 dernières années? Pourquoi le nombre d'équipes à travers le monde investies dans la recherche sur les plastiques a-t-il crû également de manière exponentielle ? Pourquoi une majorité de travaux est-elle issue du monde des sciences de la nature et se focalise-t-elle sur le compartiment océanique et non sur le compartiment continental où pourtant ils sont majoritairement produits ? Pourquoi de plus en plus de travaux s'intéressent-ils aux micro- puis nanoplastiques, alors qu'en masse les rejets sont majoritairement de grande taille? Pourquoi, dans ce qui est devenu une grande question de société, les sciences humaines et sociales n'ont-elles qu'une implication très marginale? Voilà les principales questions auxquelles ce regard croisé s'efforce de proposer des éléments de réponses et de réflexion, au travers de l'expérience d'une équipe interdisciplinaire, investie dans la recherche sur les plastiques depuis presque 10 ans.
\end{abstract}

Mots-clés : environnement / risques / développement durable / épistémologie / déchets plastiques / interdisciplinarité

\begin{abstract}
The forgotten continent. Light and shadows of research on the dispersal of plastics. In about ten years, global contamination by plastics has become a worldwide issue involving many categories of stakeholders and particularly the scientific community. Over this period, the number and production of these scientific teams has grown exponentially, giving scientific and media notoriety to many researchers. This surprising growth raises, however, many questions for number of actors involved in this research. In this article, a multidisciplinary group of researchers from the human and social sciences (socioanthropology) and the natural sciences (chemistry, Earth sciences, environmental sciences) offers its analysis of this phenomenon through its experience. The article successively explores the reasons why a) most of the work ever done is focusing on the ocean environment, while the contamination is mainly landbased, b) work is increasingly focusing on micro- and nano-plastics, while the masses discharged are mostly in the form of macro-litter, c) there is a need to highlight ecotoxicological or toxicological hazards as though the global contamination by artificial compounds was not in itself a major issue, and last d) the NGOs contribute so much in research on plastics. It also points out the major methodological difficulties when studying plastic leakage into the environment from their sources to pathways and sinks. The almost absence of the human sciences from this debate is discussed. The article concludes on the need for a much more systemic approach to better organize plastics research.
\end{abstract}

Keywords: environment / hazard / sustainable development / epistemology / plastic litter / interdisciplinarity

\footnotetext{
*Auteur correspondant : bruno.tassin@enpc.fr
} 


\section{Introduction}

Où faudrait-il vivre pour ignorer la question de la dissémination des plastiques dans l'environnement? Le sujet, fort présent dans les médias depuis quelques années, est souvent accompagné d'images spectaculaires et dramatiques. La presse fait écho, autant qu'elle participe, à la multiplication des initiatives prétendant apporter des solutions au problème. La lutte contre la pollution plastique mobilise également les élus et les administrations qui appuient les recherches scientifiques et planchent sur des évolutions réglementaires. Sensibles à la question, les collectivités locales se montrent aujourd'hui souvent prêtes à financer des dispositifs pour éliminer les microplastiques (particules inférieures à $5 \mathrm{~mm}$ ) dont sont chargées les eaux rejetées dans les cours d'eau par les stations d'épuration ou par les déversoirs d'orage. L'Union européenne et l'État français se positionnent également sur cette question (Directive " plastique à usage unique », $2019^{1}$; loi anti-gaspillage et économie circulaire, $2020^{2}$ ). Par ailleurs, les associations à vocation environnementale qui organisent des ramassages de déchets plastiques, qui génèrent des données et construisent des cartes, qui font de la sensibilisation auprès du grand public ou du lobbying auprès des élus, sont fort nombreuses. La «start-up nation » se mobilise aussi à sa manière, pour inventer des systèmes de repérage et de nettoyage plus ou moins intelligents et automatisés, tout en insistant sur la responsabilisation individuelle. Les industriels cherchent des solutions technologiques pour répondre aux préoccupations publiques et éviter d'être tenus pour responsables des dégâts environnementaux qu'engendrent les plastiques qu'ils produisent et distribuent. Chacun, c'est-à-dire chaque usager, chaque citoyen, chaque consommateur, entend les injonctions de la «transition écologique» et dans ce cadre est invité à trier ses déchets (Jacqué, 2003 ; Rumpala, 1999), à ne pas les jeter dans la nature et à s'orienter vers les produits vendus en vrac. Des pans entiers de la société sont donc mobilisés par la problématique des débris plastiques. Dans ce contexte, le monde de la recherche est également impliqué. Les publications se multiplient et certaines proposent des connaissances de plus en plus précises et de plus en plus consistantes (Gasperi et al., 2018).

\footnotetext{
${ }^{1}$ Directive 2019/904 du Parlement européen et du conseil du 5 juin 2019 relative à la réduction de l'incidence de certains produits en plastique sur l'environnement, https://eur-lex. europa.eu/eli/dir/2019/904/oj.

${ }^{2}$ Loi n $^{\circ} 2020-105$ du 10 février 2020 relative à la lutte contre le gaspillage et à l'économie circulaire, Journal officiel, 11 février 2020, 35, https://www.legifrance.gouv.fr/eli/loi/ 2020/2/10/TREP1902395L/jo/texte.
}

La thématique de la lutte contre la pollution plastique, qu'il s'agisse d'élaborer de nouveaux matériaux ou de limiter leur dissémination dans l'environnement, constitue une belle occasion pour examiner dans un contexte de crise environnementale, la manière dont les sciences s'emparent d'un sujet nouveau, la manière dont elles le traitent et l'investissent en fonction de leurs traditions expérimentales, de leurs relations avec le monde social, des valeurs, des stratégies et des communautés de chercheurs. Ce sujet est d'abord une invitation au croisement des approches entre sciences de la nature et sciences sociales. Pour ces dernières, les débris plastiques sont des objets ayant eu une vie sociale et ayant une dimension symbolique - d'abord comme support du progrès puis comme symptômes de l'impact des activités humaines sur les milieux (Monsaingeon, 2017). Ce sont aussi des matières qui en passant de nos lieux habités aux milieux naturels remettent en cause les représentations de la nature dominantes dans les sociétés occidentales (Descola, 2005; Blot et Désert, 2020). Pourtant le problème de la présence des plastiques dans l'environnement, qui connaît un immense intérêt du côté des sciences de la nature, reste assez ignoré des sciences sociales.

Le panorama des connaissances construites récemment sur la dissémination et sur l'impact des plastiques présente un fort contraste entre des terrains et des sujets qui captent les attentions et des domaines qui restent encore très peu explorés. En accord avec le battage médiatique autour des plastiques, le milieu océanique semble concentrer l'essentiel des travaux, et cela qu'ils émanent du monde scientifique ou qu'ils soient portés par les associations mobilisées sur le sujet. Pour les chercheurs, les logiques de la production scientifique et de son évaluation, celles de la recherche de financements, sont sans aucun doute à l'œuvre dans ces orientations qui privilégient les milieux océaniques et les microplastiques, voire plus récemment les nanoplastiques. Travailler à ces échelles permet aux chercheurs de faire des recherches passionnantes mobilisant des équipements coûteux, de mener des expéditions lointaines et de publier des résultats parfois spectaculaires. Du côté des associations qui s'activent en réaction à la dégradation des milieux, les représentations de la nature pèsent dans l'importance accordée aux océans et aux littoraux, comme si ces milieux étaient plus naturels que d'autres. Les associations qui subissent comme le monde scientifique un fort tropisme océanique, ont commencé par se concentrer sur les débris visibles qui ont un impact esthétique important, avant de s'intéresser aux particules de plus petite taille. Les macroplastiques (déchets supérieurs à $5 \mathrm{~mm}$ ) sont évidemment les plus accessibles quand on ne dispose pas d'un appareillage technologique complexe et coûteux. 
Dans ce paysage de connaissances, les données sur l'amont du phénomène, c'est-à-dire sur les sources de dissémination des plastiques, sont encore rares: on sait bien plus de choses sur ce qui est invisible et au milieu des océans que sur ce que nous avons presque sous les yeux au quotidien. L'étude des sources, qui implique de s'intéresser aux dimensions sociales, économiques, métaboliques du phénomène, pose des difficultés méthodologiques auxquelles sont peu habitués les chercheurs des sciences de la nature et les militants des associations. Mais le sujet est aussi marginal du côté des sciences sociales enclines à traiter des interactions sociales, des institutions et des systèmes de gestion bien plus que des objets échappant au contrôle des humains. Par ailleurs, les représentations courantes, expliquant la présence de déchets dans l'environnement par les comportements individuels inadaptés, les fameuses « incivilités », occupent le champ et laissent peu de place aux analyses que pourraient produire les sciences sociales. Les obstacles à la construction de connaissances globales et interdisciplinaires résultent de la possibilité qu'ont les sciences de la nature d'étudier la dissémination des plastiques sans révolutionner leurs méthodes expérimentales habituelles alors que les sciences sociales sont, avec ce sujet, confrontées aux limites fixées par leur tradition disciplinaire et par une demande faible.

\section{Du côté des sciences de la nature: tropisme océanique et quête de l'infiniment petit}

\section{Un intérêt marqué pour les océans}

Parallèlement aux nombreuses inquiétudes que suscite la présence des plastiques dans l'environnement, la recherche s'est développée depuis une dizaine d'années à un rythme accéléré (Gasperi et al., 2018). Les publications sur le thème sont si fréquentes aujourd'hui qu'il est devenu impossible pour un scientifique de lire l'ensemble de la bibliographie. La très grande majorité de l'activité de recherche se concentre sur une évaluation de la quantité des plastiques dans les masses d'eau marines, sur leur devenir et leur impact, même si elle s'intéresse de plus en plus au milieu continental. En 2012, ce milieu représentait $1 \%$ du nombre total des publications sur les plastiques dans le domaine des sciences de l'environnement et, en $2020,15 \%{ }^{3}$.

\footnotetext{
3 L'interrogation de la base de données bibliographiques Scopus pour l'année 2020, avec la requête sur les mots-clés «plastic» ou «microplastic» ou «nanoplastic», limitée au champ des sciences de l'environnement, renvoie, en incluant le mot-clé «freshwater», sur 179 articles contre 1174 avec le mot-clé «ocean».
}

Tout a commencé en 1972 lorsque Carpenter et Smith publièrent la première étude faisant état de la présence de particules plastiques dans la mer des Sargasses (Carpenter et Smith, 1972). Depuis, c'est principalement par la métaphore du $« 7^{\mathrm{e}}$ continent $»$, heureuse traduction en français du «great Pacific garbage patch » médiatisé en 1997 par Charles Moore, que la question a gagné la sphère publique en France (Monsaingeon, 2016). Les études montrent une pollution plastique ubiquiste, de plus en plus intense avec des « garbage patch» identifiés dans l'ensemble des grands gyres océaniques et dans la Méditerranée (Barnes et al., 2009; Law et al., 2010; Sebille et al., 2015; Eriksen et al., 2014; Ryan, 2014 ; Cózar et al., 2015 ; Jambeck et al., 2015 ; Lebreton et al., 2018). Parallèlement, la production croît de manière exponentielle : en 2019 , ce sont 450 millions de tonnes de plastique et de fibres plastiques qui ont été produites (PlasticsEurope, 2020 ; Engelhardt, 2020) et la pandémie de Covid-19 ne l'a qu'à peine ralenti : 367 millions de tonnes (hors fibres) en 2020 contre 368 en 2019 (PlasticsEurope, 2021). Entre 1 et 10 millions de tonnes de ces plastiques se répandraient chaque année dans les océans avec une grande disparité géographique des émissions (Jambeck et al., 2015; Lebreton et al., 2017; Schmidt et al., 2017; Meijer et al., 2021 ${ }^{4}$ ). D'ici 2050, Wilcox et al. (2015) prédisent que $99 \%$ des espèces d'oiseaux marins auront dû affronter des macroplastiques avec tous les risques de mortalité par étouffement, emmêlement ou famine que cela comporte. Thiel et al. (2018) font un constat similaire dans le Pacifique Sud concernant 5 espèces de tortues marines, 20 espèces de poissons et 19 espèces de mammifères. Pire encore, plus de $92 \%$, en nombre, du plastique dans les océans seraient des microplastiques (Eriksen et al., 2014; Lebreton et al., 2018) dont les effets écotoxicologiques potentiels sur les organismes aquatiques sont nombreux (Chae et An, 2017; Anbumani et Kakkar, 2018).

\section{À la conquête de l'infiniment petit}

Après avoir essentiellement étudié les microplastiques, les recherches s'intéressent depuis 2016 à l'infiniment petit avec une thématique émergente: les nanoplastiques (particules de taille inférieure à $1 \mu \mathrm{m}$ ). Cette orientation s'accompagne d'une sorte de course à l'armement technologique avec des machines dont la complexité n'a d'égal que leur prix pour le plus grand bonheur des industriels du matériel analytique de laboratoire. Seules les équipes capables de suivre technologiquement et financièrement peuvent donc s'engager dans cette voie. Les sciences expérimentales

\footnotetext{
4 Toutefois, des travaux très récents évoquent des flux à l'océan plus faibles d'un à deux ordres de grandeur (par exemple, Weiss et al., 2021).
} 
fournissent aujourd'hui d'extraordinaires résultats qui sont repris par les médias: par exemple, la mise en évidence de la présence des nanoplastiques dans l'Atlantique Nord (Ter Halle et al., 2017) mis en avant dans la presse (La Dépêche du Midi, Davodeau, 2017). Mais parce qu'il mobilise des équipements de haute technologie et parce qu'il n'implique pas de révolution des protocoles scientifiques, le travail sur les microplastiques et les nanoplastiques est souvent facile à concevoir et il permet, sans prendre trop de risque, de produire des résultats dont la valeur sera facilement reconnue. L'orientation des recherches est polarisée par les logiques de reconnaissance et d'évaluation des chercheurs. Travailler sur cet infiniment petit, c'est se donner davantage de chance de faire des découvertes, de publier, d'être cité, d'améliorer son «h-index» et d'enrichir son $\mathrm{CV}^{5}$. Les résultats peuvent aussi être insolites, comme le fait de trouver des nanoplastiques dans les excréments humains (Schwabl et al., 2019), ou très inquiétants, quand on constate leur présence dans le placenta (Ragusa et al., 2021). Ils ont alors des retombées médiatiques immédiates ${ }^{6}$. L'audience dans la société, qui complète la reconnaissance purement scientifique des travaux, est une autre forme de valorisation à laquelle le monde de la recherche semble de plus en plus sensible. Certes, les chercheurs sont loin d'être tous obsédés par la reconnaissance, mais l'innovation scientifique n'est pas indépendante des conditions de construction et de diffusion des connaissances qui sont socialement déterminées (Latour, 1993). On peut ainsi faire l'hypothèse que l'effervescence scientifique autour de ces questions s'explique en partie par la recherche de la reconnaissance des pairs, qui constitue un atout pour construire une carrière et pour obtenir des moyens et des équipements. La fuite en avant vers les micro- et nanoparticules doit donc autant sa force au mode d'évaluation et de financement des recherches qu'à des justifications proprement scientifiques.

\footnotetext{
5 Le $h$-index vise à mesurer l'impact cumulatif de la productivité d'un chercheur en prenant à la foi en compte le nombre de citations que son travail a reçues et la quantité de publications.

${ }^{6}$ Des indicateurs comme les altmetrics calculent l'impact sur Internet des travaux de recherche en se basant sur leur diffusion et les actions et interactions qu'ils engendrent sur les réseaux sociaux, les blogs, dans la presse, les bases de données bibliographiques, etc. (Dupré et al., 2016). Le papier sur la présence des microplastiques sur les sommets pyrénéens (Allen et al., 2019) a un score d'altmetric de 1939 (au 17 janvier 2022), quand celui de Ter Halle et al. (2017) sur les nanoplastiques dans les océans est de 55 à la même date. La différence de résonance médiatique s'explique moins par la portée scientifique du propos que par son contenu plus facile à percevoir et à appréhender.
}

Il est aussi assez probable qu'un certain nombre de chercheurs aient la volonté de travailler sur ce thème en raison de leurs convictions écologiques. Le rôle des valeurs dans le choix des sujets de recherche est identifié depuis bien longtemps (Weber, 1965 [1904]), mais nous sommes sans doute dans une configuration nouvelle avec l'anthropisation des milieux : les scientifiques sont investis d'un rôle d'experts, de témoins et de lanceurs d'alerte, comme en témoignent les interviews pour lesquelles ils sont fréquemment sollicités. Le fait que les plastiques océaniques soient identifiés comme risque global au même titre que la crise climatique ou la $6^{\mathrm{e}}$ extinction de masse des espèces, et la mobilisation de pans entiers de la société autour de cette question (Gasperi et al., 2018) rendue visible par son hypermédiatisation, facilitent également l'obtention de financements nécessaires à ces recherches (par exemple les financements de l'Agence nationale de la recherche [ANR] ou de l'Union européenne, qui ciblent explicitement ces recherches).

\section{La recherche des risques potentiels des microplastiques et des nanoplastiques}

D'un point de vue écotoxicologique, l'étude des microet nanoplastiques est porteuse de sens du fait de l'augmentation des risques, supposés ou avérés, à mesure que les particules deviennent de plus en plus petites. Les études abondent sur les effets néfastes des plastiques sur les organismes aquatiques à tous les niveaux de la chaîne trophique (Chae et An, 2017; Anbumani et Kakkar, 2018). Par exemple, les micro- et nanoplastiques ont la capacité de transférer des micropolluants absorbés sur leur surface aux microorganismes aquatiques qui les ingèrent. De l'étude des microplastiques a émergé la découverte d'un nouvel écosystème : la plastisphère (Zettler et al., 2013). Ce terme fait référence à l'abondance d'organismes de natures diverses (par exemple autotrophes, hétérotrophes, prédateurs) interagissant entre eux au sein du substrat plastique. La prolifération de ces organismes, dont certains peuvent être pathogènes ou encore invasifs, pose un certain nombre de problèmes dans la mesure où leur substrat, non biodégradable, leur confère une capacité de dissémination inédite (Maso et al., 2003). Cependant, beaucoup de questions restent en suspens concernant l'impact de l'ingestion des micro- et nanoplastiques sur la santé humaine via l'eau, l'air ou l'alimentation. Davantage que les résultats, ce sont aujourd'hui l'incertitude et le niveau du risque qui justifient que les recherches soient poursuivies, car de grandes incertitudes s'accompagnent de grands risques.

\section{Les sciences humaines et sociales en retrait}

Un frein à la recherche sur les fuites de plastiques est constitué par la posture des chercheurs en sciences 
sociales qui ne s'emparent guère de la question. La disproportion entre les centaines de chercheurs en sciences de la nature qui travaillent sur ce thème et la petite poignée de ceux qui, en sciences sociales, s'en saisissent est flagrante. Ces derniers ont longtemps souffert d'une position de handicap épistémologique sur ce genre de thème parce que l'objet même de ces sciences est le «social» (Barbier, 2002; Fabiani, 2017). Certes, les objets physiques sont étudiés par les sociologues et les anthropologues en tant que porteurs de relations et d'interactions sociales (Veblen, 1970 [1901]; Mauss, 2007 [1925]; Appadurai, 1986), et la question des relations entre les humains et leurs environnements matériels inspire les sciences sociales depuis fort longtemps (Demeulenaere, 2017), mais développer une sociologie ou une anthropologie des plastiques perdus dans l'environnement constitue un défi scientifique important (Blot, 2015). Les sciences sociales vont être assez peu armées pour appréhender ces objets dispersés autrement que par les réactions qu'ils suscitent. Ainsi à quelques exceptions près, les travaux actuels s'intéressent davantage aux collecteurs et aux recycleurs de déchets sauvages, aux militants qui dénoncent les atteintes à l'environnement qu'ils constituent qu'à ces déchets et aux processus qui leur confèrent ce statut. La «stigmatisation académique » (Le Dorlot, 2004 ; Corteel et Le Lay, 2011) des recherches sur les déchets s'estompe et aujourd'hui la demande institutionnelle d'études sur le recyclage et le réemploi et sur le traitement des déchets ordinaires est assez conséquente. En revanche, les déchets sauvages restent des objets délaissés par la sociologie et par l'anthropologie. La géographie de son côté possède une ancienne et solide tradition de recherche sur les déchets (Gouhier, 1984) et sur leurs flux mais elle ne s'est pour le moment pas encore mobilisée pour éclairer ce qui mène les objets des espaces fortement anthropisés à ceux qui le sont beaucoup moins. Sur la problématique des plastiques perdus dans l'environnement, les sciences sociales se retrouvent donc dans une situation quasiment opposée à celle des sciences de la nature. Là où les chercheurs des sciences de la nature peuvent espérer des financements et une bonne reconnaissance sans modifier radicalement leurs traditions expérimentales, les chercheurs des sciences sociales doivent surmonter des obstacles épistémologiques et méthodologiques sans pouvoir, pour le moment, espérer des soutiens et des gratifications significatives.

Les sciences sociales sont souvent interpellées par des collectivités territoriales et par des associations agissant dans le secteur des déchets sauvages, mais c'est pour répondre à des questions qui relèvent plus de la gestion des populations par des opérations de police ou d'éducation (comment sanctionner ou comment sensibiliser) et non pour apporter des données alimentant la connaissance du phénomène. Ainsi, ce sont les stéréotypes sociaux pointant en premier lieu les «incivilités » qui nourrissent les discours. Ce sont alors les «autres» qui sont désignés comme responsables : ceux qui feraient preuve d'une faible sensibilité écologique, qui ne seraient pas éduqués, ou qui seraient sales (Blot, 2015; Blot et Désert, 2020). Ajoutons que des associations financées par les industries de l'emballage participent à la diffusion de l'idée selon laquelle la dissémination des plastiques est un problème de propreté individuelle (par exemple, Gestes propres, en France, Keep America Beautiful, aux États-Unis). Or, parmi l'ensemble des plastiques retrouvés dans l'environnement, nul ne sait aujourd'hui quelle est la proportion imputable à des comportements individuels inappropriés. Bien qu'elles ne soient ni neutres, ni satisfaisantes, ces explications sur l'origine des débris plastiques jouent leur rôle, occupent l'espace et semblent freiner le développement de recherches sur l'amont du phénomène ou alors les aiguillent sur une toute petite partie du problème. La politique de croissance, solidaire d'un modèle économique basé sur la consommation, et les intérêts d'une «filière plastique » représentant en Europe plus d'un million et demi d'emplois et un chiffre d'affaires de 350 milliards d'euros ne sont par conséquent jamais questionnés (PlasticsEurope, 2020). Ainsi les financements publics alloués par l'Agence de l'environnement et de la maîtrise de l'énergie (Ademe) en 2018 ont concerné les "déchets sauvages" définis comme «déchets abandonnés dans l'environnement» avec pour objectifs de sensibiliser et de prévenir ces dépôts en s'adressant aux usagers, voire de valoriser ces déchets une fois retournés dans la gestion classique des déchets. Cette perspective hygiéniste vise avant tout à garantir la propreté publique, alors que la dissémination des plastiques mériterait une approche bien plus globale et débarrassée de toute coloration morale et de toute idéologie.

\section{Du côté des associations: macrodéchets et océans}

\section{Des connaissances locales fines sur les macroplastiques}

Les associations et les $\mathrm{ONG}$ participent également à la construction de connaissances en s'orientant majoritairement elles aussi sur les littoraux et sur les fleuves qui alimentent les océans en plastiques (Schmidt et al., 2017). Parce que les macroplastiques sont particulièrement visibles dans les paysages, et parce qu'ils sont des microplastiques en devenir par la fragmentation (Eriksen et al., 2014; Lebreton et al., 2018), les associations ont commencé à s'y intéresser. Celles qui organisent des collectes de déchets sauvages et qui se sont emparées de 
la question dès les années 1990-2000, sont devenues des acteurs incontournables, notamment parce qu'elles ont su trouver des relais dans les médias et auprès des pouvoirs publics. Les microplastiques restent généralement hors de leur portée : par manque de moyens ou par volonté de «nettoyer la nature», ces associations (SOS Mal de Seine; SeaMer; NatureLibre; Ansel; etc.) se sont concentrées sur les macrodéchets en général, dont les macroplastiques (Poitou, 2003; Bruge et al., 2018) qui représentent la majeure partie de la masse des plastiques visibles en mer. Pour des associations ayant une vocation d'alerte ou de sensibilisation, les macroplastiques permettent la diffusion d'images assez spectaculaires. Leur impact sur les paysages ou sur les grands animaux est manifeste, comme en témoignent les photographies des mammifères marins aux tubes digestifs pleins de sacs plastiques, qui se retrouvent épisodiquement dans la presse et sur les affiches destinées au grand public (Monsaingeon, 2016). Les macroplastiques sont donc des bons sujets pour les médias. Ils permettent par ailleurs d'organiser des collectes bénévoles ayant pour objectif de nettoyer les plages et de sensibiliser le public (Blot, 2016). Pour les politiques, ils donnent l'occasion de répondre à une demande sociale, sans avoir à rechercher de solution à l'échelle mondiale comme pour la crise climatique, et, dans une première approche, sans risque de remettre en cause le modèle économique dominant en focalisant l'espace des solutions autour de stratégies qui ne s'attaquent aucunement à la source du problème. Le tout recyclage, par exemple, qui semble être la pierre d'angle de l'économie circulaire se développant actuellement, n'a pas pour objectif de réduire la production et la circulation des plastiques. $\mathrm{Au}$ contraire, la simplification du geste de tri promu par les autorités via CITEO vise à augmenter la collecte d'emballages plastiques pour développer des filières de recyclage économiquement viables au sein d'une économie de marché ${ }^{7}$. Dès lors, il est difficile d'imaginer une diminution significative du volume qui se perd dans l'environnement.

Certaines associations disposent maintenant de données locales très fines. Malheureusement, l'hétérogénéité de ces données rend leur compilation délicate. Tout l'enjeu est donc de développer des méthodes communes qui permettent d'exploiter et de comparer des bases de données issues des collectes bénévoles. Les associations bénéficient du soutien des pouvoirs publics pour travailler dans ce sens avec par exemple la mise en place depuis 2018 d'un atelier « déchets marins » et depuis 2020 d'un atelier «surveillance macrodéchets cours d'eau » au ministère de la Transition écologique. Ces ateliers constituent des espaces de discussion visant à faire émerger des idées

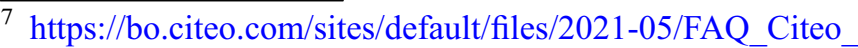
Simplification_du_tri_20210526.pdf.
}

d'actions concrètes en concertation avec l'État, ses établissements publics en charge de la mer et les associations de protection de l'environnement, au sein du Comité France Océan, sous l'autorité du Premier ministre ${ }^{8}$. À l'échelle européenne, un protocole de collecte commun, dont la dernière mise à jour remonte à $2021^{9}$, a été mis en place. Ce protocole vise à mieux caractériser les déchets plastiques échoués sur les plages d'Europe de l'Ouest, ainsi que leurs sources, afin d'établir un diagnostic de la pollution liée aux macrodéchets, dont les macrodéchets plastiques. Actuellement, il se développe aussi pour les fleuves et rivières avec des collectes de déchets le long de linéaires de berge. Malheureusement, sa lourdeur et la difficulté d'accéder aux berges, par rapport au littoral, découragent certaines bonnes volontés. Pour lever certains de ces obstacles, la France a développé la plateforme Zéro déchet sauvage ${ }^{10}$ qui permet de valoriser des données de collectes en fonction de différents niveaux de protocoles afin d'inclure le plus grand nombre d'initiatives. Mais des suivis pérennes et de qualité, utilisables pour la recherche scientifique et pour une orientation éclairée des politiques publiques, appellent à la professionnalisation de la surveillance des macrodéchets diffus dans différents compartiments de l'environnement.

\section{L'océan : le territoire vierge par excellence}

L'intérêt pour les océans peut s'expliquer par le fait qu'ils constituent effectivement les endroits où finissent et où s'accumulent les débris plastiques; particulièrement dans les fonds marins (Galgani et al., 2000; Kammann et al., 2018; Maes et al., 2018). Mais ils sont aussi des ressources importantes pour l'imaginaire occidental: les océans correspondent aux représentations dominantes d'une nature immense et en partie inexplorée (Blot et Désert, 2020) ; là plus qu'ailleurs, la présence de plastiques semble l'abîmer. Les océans offrent aussi un cadre esthétique de grande qualité qui se prête parfaitement à la fabrication de belles images et à la mise en scène de la chasse au plastique (Monsaingeon, 2016). Ils se prêtent à toutes sortes d'investissements et d'aventures: des expéditions, des opérations de nettoyage sur le littoral qui sont bien plus aisées que dans d'autres milieux parce que les plastiques sont «propres» et le terrain souvent dégagé, ou encore des projets de nettoyage à grande échelle comme celui du désormais célèbre Boyan Slat « The Ocean Cleanup ». En proposant des solutions curatives focalisées sur la fin de vie du plastique, les opérations de nettoyage oblitèrent l'amont du processus et les choix politiques de production, de distribution et les injonctions de consommation (Blot et Désert, 2019).

\footnotetext{
${ }^{8}$ https://www.gouvernement.fr/comite-france-ocean.

9 https://mcc.jrc.ec.europa.eu/main/dev.py? $\mathrm{N}=41 \& \mathrm{O}=459$.

10 https://www.zero-dechet-sauvage.org.
} 


\section{Le continent oublié}

\section{Un grand déficit de travaux sur l'amont du processus}

Le problème de cette configuration est qu'elle laisse dans l'ombre une bonne partie des phénomènes à l'origine de la dissémination des plastiques: les travaux qui l'abordent sont encore rares ou très localisés (Lechner et al., 2014; Dris et al., 2015; Van der Wal et al., 2015; Lahens et al., 2018). Les manières dont ils rejoignent les océans restent mal connues et mal quantifiées. La réalisation d'un diagnostic fiable concernant les flux de plastique depuis les continents est pourtant le préalable à toute réglementation visant à réduire efficacement cette pollution. La tâche est ardue : la circulation à l'échelle mondiale des plastiques, en particulier ceux qui sont usagés, est soumise à des évolutions fréquentes; les voies de transfert dans l'environnement sont multiples; les fuites qui résultent de ces circulations ne sont ni bien identifiées ni bien quantifiées; etc. Certes, des efforts sont entrepris (Lebreton et al., 2017; Schmidt et al., 2017; Meijer et al., 2021). Néanmoins, c'est toujours l'impact sur les océans qui est l'objectif final.

\section{Des difficultés méthodologiques réelles}

Outre que l'étude des macroplastiques ne fait pas appel à de la «belle science», des beaux objets et rarement à des équipements techniques sophistiqués, elle est confrontée à de réelles difficultés méthodologiques (Van der Wal et al., 2015). Ces difficultés tiennent au fait que les plastiques qui échappent à la maîtrise des systèmes techniques représentent un volume très faible par rapport au volume global produit par les industries. Ces fuites de plastique sont par ailleurs diffuses, multiples et aux causes variées. Elles peuvent aussi bien être liées à la faune qui s'attaque aux poubelles, qu'au vent qui emporte des plastiques légers, qu'au coup de scie dans un bloc d'isolant sur un chantier de bâtiment, qu'aux fuites des dispositifs de collecte des poubelles ou de transport des matières premières. Les crues et les tempêtes sont également un facteur de dissémination. Ces événements, bien que ponctuels, produisent un nombre considérable de déchets dont une bonne partie est en plastique, mais l'estimation de leur contribution à la pollution globale est très difficile. Pour ajouter à la complexité du phénomène, les fuites sont présentes à tous les stades de vie d'un produit, de sa production jusqu'à sa déchéance en passant par son transport et par sa consommation. Les granulés plastiques industriels - matière première de quelques millimètres de diamètre qui jonchent certaines plages et berges de rivières, sont un très bon exemple de perte de plastique lors du processus de production et de transport (Karlsson et al., 2018).

\section{Conclusion: vers une écologie globale des plastiques?}

Les difficultés méthodologiques et épistémologiques, les possibilités différentielles de valoriser les recherches et l'intérêt privilégié accordé aux océans pour des motifs tenant en grande partie aux représentations occidentales de la nature et à la facilité de les mettre en scène, sont autant de raisons de la disproportion manifeste entre ce que l'on sait de la présence des plastiques dans les milieux marins et ce que l'on sait de leur origine. Ce panorama des connaissances permet d'identifier les freins au développement d'une écologie globale des plastiques, c'est-à-dire d'envisager une perspective qui prenne en compte l'ensemble de leur environnement en intégrant autant les "choses de la nature» que les «choses sociales» tels que les chercheurs, les militants associatifs, les industriels, les institutions et le personnel politique, les normes, les usages, les règlements et les dispositifs techniques. Les pratiques de chacun vis-à-vis du plastique et sa perception sont aussi importantes à saisir. Nous lançons un appel à investiguer dans les zones les moins explorées, comme les bords de route ou les voies de chemin de fer, qui sont étonnamment les plus proches géographiquement et celles qui nécessitent le moins d'équipement lourd et coûteux. C'est aussi une invitation à appréhender de manière différente, plus complète et sans doute plus lucide, les solutions proposées pour faire face à la dissémination des plastiques. Le tropisme océanique n'explique-t-il pas en grande partie le développement de solutions de nettoyage en aval, voire de recyclage des plastiques collectés dans les océans, au détriment des initiatives qui permettraient de freiner drastiquement le volume de fuite en amont? La passion pour les micro- et les nanoplastiques n'est-elle pas, elle aussi, responsable de l'apparition de solutions technologiques qui laissent entendre qu'un plastique recyclé ou biosourcé serait une solution alors qu'il est tout aussi nocif que n'importe quel autre s'il se retrouve dans l'environnement? Et d'ailleurs, quand bien même il serait vraiment inoffensif, sa présence dans les milieux naturels est-elle acceptable?

Nous pensons que la faiblesse actuelle des connaissances sur l'origine des plastiques dans les milieux océaniques est l'une des raisons pour lesquelles la réduction globale de leur production ne fait presque jamais partie des solutions envisagées. Ainsi l'orientation des recherches vers l'aval et vers l'infiniment petit pourrait bien participer à la diffusion de l'idée que la présence de plastique dans les océans est une sorte de fatalité, une donnée environnementale comme une autre. $\mathrm{Au}$ contraire, une réorientation des recherches sur 
l'amont des processus qui conduisent les ressources premières à se transformer en déchet, a fortiori en déchet « sauvage », pourrait mener à des solutions novatrices et systémiques à même de répondre aux autres enjeux du siècle, tels que ceux posés par les micropolluants ou le réchauffement climatique.

\section{Références}

Allen S., Allen D., Phoenix V.R., Le Roux G., Durántez Jiménez P., Simonneau A., Binet S., Galop D., 2019. Atmospheric transport and deposition of microplastics in a remote mountain catchment, Nature Geoscience, 12, 339-344, https://doi.org/10.1038/s41561-019-0335-5.

Anbumani S., Kakkar P., 2018. Ecotoxicological effects of microplastics on biota: a review, Environmental Science and Pollution Research, 25, 14373-14396, https://doi.org/ 10.1007/s11356-018-1999-x.

Appadurai A. (Ed.), 1986. The social life of things. Commodities in cultural perspective, Cambridge, Cambridge University Press.

Barbier R., 2002. La fabrique de l'usager. Le cas de la collecte sélective des déchets, Flux, 48-49, 2-3, 35-46, https://doi. org/10.3917/flux.048.0035.

Barnes D.K.A., Galgani F., Thompson R.C., Barlaz M., 2009. Accumulation and fragmentation of plastic debris in global environments, Philosophical Transactions of the Royal Society B, 364, 1985-1998, https://doi.org/10.1098/ rstb.2008.0205.

Blot D., 2015. À qui sont ces déchets? Oppositions sociales et conflits de territoires dans le traitement des déchets sauvages, in Durand M., Djellouli Y., Noarine C. (Eds), Gestion des déchets. Innovations sociales et territoriales, Rennes, Presses universitaires de Rennes, 229-240.

Blot D., 2016. Nettoyer les plages pour habiter la nature, in Robert S., Melin H. (Eds), Habiter le littoral. Enjeux contemporains, Aix-en-Provence, Presses universitaires de Provence/Presses universitaires d'Aix-Marseille, 173-184.

Blot D., Désert J., 2019. Nettoyer la nature, est-ce vraiment une bonne idée?, The Conversation, 19 décembre, https:// theconversation.com/nettoyer-la-nature-est-ce-vraimentune-bonne-idee-128151.

Blot D., Désert J., 2020. Discours sur la mer, résistance des plastiques, in Bouvet Y., Page-Jones K. (Eds), Discours sur la mer. Résistance des pratiques et des représentations, Rennes, Presses universitaires de Rennes, 135-150.

Bruge A., Barreau C., Carlot J., Collin H., Moreno C., Maison P., 2018. Monitoring litter inputs from the Adour river (Southwest France) to the marine environment, Journal of Marine Science and Engineering, 6, 1, 24, https://doi.org/ 10.3390/jmse6010024.

Carpenter E.J., Smith K.L., 1972. Plastics on the Sargasso sea surface, Science, 175, 4027, 1240-1241, https://doi.org/ 10.1126/science. 175.4027.1240.

Chae Y., An Y.-J., 2017. Effects of micro- and nanoplastics on aquatic ecosystems: current research trends and perspectives, Marine Pollution Bulletin, 124, 2, 624-632, https://doi.org/10.1016/j.marpolbul.2017.01.070.

Corteel D., Le Lay S., 2011. Les travailleurs des déchets, Toulouse, Érès.

Cózar A., Sanz-Martín M., Martí E., González-Gordillo J.I., Ubeda B., Gálvez J.Á., Irigoien X., Duarte C.M., 2015. Plastic accumulation in the Mediterranean sea, PLOS ONE, 10, 4, e0121762, https://doi.org/10.1371/journal. pone. 0121762 .

Davodeau B., 2017. Alexandra Ter Halle, chimiste: «Le plastique rejeté dans l'océan se fractionne en nanoparticules », Ladepeche.fr, $1^{\text {er }}$ décembre, https://www.ladepeche. fr/article/2017/12/01/2695923-le-plastique-rejete-dans-1ocean-se-fractionne-en-nanoparticules.html.

Demeulenaere E., 2017. L'anthropologie au-delà de l'anthropos. Un récit par les marges de la discipline, in Blanc G., Demeulenaere E., Feuerhahn W. (Eds), Humanités environnementales. Enquêtes et contre-enquêtes, Paris, Éditions de la Sorbonne, 43-73, https://doi.org/10.4000/ books.psorbonne. 84330 .

Descola P., 2005. Par-delà nature et culture, Paris, Gallimard.

Dris R., Imhof H., Sanchez W., Gasperi J., Galgani F., Tassin B., Laforsch C., 2015. Beyond the ocean: contamination of freshwater ecosystems with (micro-)plastic particles, Environmental Chemistry, 12, 5, 539-550, https://doi.org/ 10.1071/EN14172.

Dupré S., Deboin M.-C., Fovet-Rabot C., 2016. Se familiariser avec les altmetrics, mesures alternatives d'impact d'une publication, Montpellier, Cirad, https://doi.org/10.18167/ coopist/0049.

Engelhardt A.W., 2020. The Fiber Year 2020-Deceleration along the textile chain, International Fiber Journal, July 16, https://fiberjournal.com/the-fiber-year-2020-decelerationalong-the-textile-chain/.

Eriksen M., Lebreton L.C.M., Carson H.S., Thiel M., Moore C. J., Borreron J.C., Galgani F., Ryan P.G., Reisser J., 2014. Plastic pollution in the world's oceans: more than 5 trillion plastic pieces weighing over 250,000 tons afloat at sea, PLoS ONE, 9, 12, e111913, https://doi.org/10.1371/journal. pone.0111913.

Fabiani J.-L., 2017. Rural, environnement, sociologie, in Hamman P. (Ed.), Ruralité, nature et environnement, Toulouse, Érès, 111-132.

Galgani F., Leaute J., Moguedet P., Souplet A., Verin Y., Carpentier A., Goraguer H., Latrouite D., Andral B., Cadiou Y., Mahe J., Poulard J., Nerisson P., 2000. Litter on the sea floor along European coasts, Marine Pollution Bulletin, 40, 6, 516-527, https://doi.org/10.1016/ S0025-326X(99)00234-9.

Gasperi J., Blot D., Tassin B., Tramoy R., 2018. Pollution plastique, retour sur une prise de conscience, The Conversation, 10 septembre, http://theconversation.com/ pollution-plastique-retour-sur-une-prise-de-conscience101541.

Gouhier J., 1984. Déchets. L'art d'accommoder les restes, Paris, Centre Georges Pompidou. 
Jacqué M., 2003. La formation de l'écocitoyen, Communications, 74, 103-116, https://doi.org/10.3406/ comm.2003.2131.

Jambeck J.R., Geyer R., Wilcox C., Siegler T.R., Perryman M., Andrady A., Narayan R., Law K.L., 2015. Plastic waste inputs from land into the ocean, Science, 347, 6223, 768771, https://doi.org/10.1126/science.1260352.

Kammann U., Aust M.-O., Bahl H., Lang T., 2018. Marine litter at the seafloor-Abundance and composition in the North Sea and the Baltic Sea, Marine Pollution Bulletin, 127, 774-780, https://doi.org/10.1016/j.marpol bul.2017.09.051.

Karlsson T.M., Arneborg L., Broström G., Almroth B.C., Gipperth L., Hassellöv M., 2018. The unaccountability case of plastic pellet pollution, Marine Pollution Bulletin, 129, 52-60, https://doi.org/10.1016/j.marpolbul.2018.01.041.

Lahens L., Strady E., Kieu-Le T.-C., Dris R., Boukerma K., Rinnert E., Gasperi J., Tassin B., 2018. Macroplastic and microplastic contamination assessment of a tropical river (Saigon River, Vietnam) transversed by a developing megacity, Environmental Pollution, 236, 661-671, https:// doi.org/10.1016/j.envpol.2018.02.005.

Latour B., 1993. Petites leçons de sociologie des sciences, Paris, La Découverte.

Law K.L., Moret-Ferguson S., Maximenko N.A., Proskurowski G., Peacock E.E., Hafner J., Reddy C.M., 2010. Plastic accumulation in the North Atlantic subtropical gyre, Science, 329, 5996, 1185-1188, https://doi.org/10.1126/ science.1192321.

Le Dorlot E., 2004. Les déchets ménagers : pour une recherche interdisciplinaire, Strates, 11, https://doi.org/10.4000/stra tes. 410 .

Lebreton L.C.M., van der Zwet J., Damsteeg J.-W., Slat B., Andrady A., Reisser J., 2017. River plastic emissions to the world's oceans, Nature Communications, 8, 15611, https:// doi.org/10.1038/ncomms 15611 .

Lebreton L., Slat B., Ferrari F., Sainte-Rose B., Aitken J., Marthouse R., Hajbane S., Cunsolo S., Schwarz A., Levivier A., Noble K., Debeljak P., Maral H., Schoeneich-Argent R., Brambini R., Reisser J., 2018. Evidence that the Great Pacific garbage patch is rapidly accumulating plastic, Scientific Reports, 8, 4666, https://doi.org/10.1038/ s41598-018-22939-w.

Lechner A., Keckeis H., Lumesberger-Loisl F., Zens B., Krusch R., Tritthart M., Glas M., Schludermann E., 2014. The Danube so colourful: a potpourri of plastic litter outnumbers fish larvae in Europe's second largest river, Environmental Pollution, 188, 177-181, https://doi.org/ 10.1016/j.envpol.2014.02.006.

Maes T., Barry J., Leslie H.A., Vethaak A.D., Nicolaus E.E. M., Law R.J., Lyons B.P., Martinez R., Harley B., Thain J.E., 2018. Below the surface: twenty-five years of seafloor litter monitoring in coastal seas of North West Europe (1992-2017), Science of the Total Environment, 630, 790-798, https://doi.org/10.1016/j.scito tenv.2018.02.245.

Maso M., Garcés E., Pagès F., Camp J., 2003. Drifting plastic as a potential vector for dispersing harmful algal bloom
(HAB) species, Scientia Marina, 67, 1, https://doi.org/ 10.3989/scimar.2003.67n1107.

Mauss M., 2007 [1925]. Essai sur le don. Forme et raison de l'échange dans les sociétés archaïques, Paris, Presses universitaires de France.

Meijer L.J.J., van Emmerik T., van der Ent R., Schmidt C., Lebreton L., 2021. More than 1000 rivers account for $80 \%$ of global riverine plastic emissions into the ocean, Science Advances, 7, 18, https://doi.org/10.1126/sciadv.aaz5803.

Monsaingeon B., 2016. Faire monde avec l'irréparable. Sur les traces des océans de plastique, Techniques \& Culture, 65-66, 1, 34-47, https://doi.org/10.4000/tc.7777.

Monsaingeon B., 2017. Homo detritus. Critique de la société du déchet, Paris, Le Seuil.

PlasticsEurope, 2020. Plastics - the Facts 2020. An analysis of European plastics production, demand and waste data, PlasticsEurope, Association of Plastics Manufacturers, https://plasticseurope.org/knowledge-hub/plastics-thefacts-2020/.

PlasticsEurope, 2021. Plastics - the Facts 2021. An analysis of European plastics production, demand and waste data, PlasticsEurope, Association of Plastics Manufacturers, https://plasticseurope.org/knowledge-hub/plastics-thefacts-2021/.

Poitou I., 2003. Les macrodéchets littoraux: une gestion publique empirique en région Provence-Alpes-Côte d'Azur, Méditerranée, 100, 17-22, https://doi.org/ 10.3406/medit.2003.3277.

Ragusa A., Svelato A., Santacroce C., Catalano P., Notarstefano V., Carnevali O., Papa F., Rongioletti M.C.A., Baiocco F., Draghi S., D’Amore E., Rinaldo D., Matta M., Giorgini E., 2021. Plasticenta: first evidence of microplastics in human placenta, Environnement International, 146, 106274, https://doi.org/10.1016/j.envint.2020.106274.

Rumpala Y., 1999. Le réajustement du rôle des populations dans la gestion des déchets ménagers. Du développement des politiques de collecte sélective à l'hétérorégulation de la sphère domestique, Revue française de science politique, 49, 4-5, 601-630, https://doi.org/10.3406/ rfsp.1999.396247.

Ryan P.G., 2014. Litter survey detects the South Atlantic 'garbage patch', Marine Pollution Bulletin, 79, 1-2, 220-224, https://doi.org/10.1016/j.marpolbul.2013.12.010.

Schmidt C., Krauth T., Wagner S., 2017. Export of plastic debris by rivers into the Sea, Environmental Science and Technology, 51, 21, 12246-12253, https://doi.org/10.1021/ acs.est.7b02368.

Schwabl P., Köppel S., Königshofer P., Bucsics T., Trauner M., Reiberger T., Liebmann B., 2019. Detection of various microplastics in human stool: a prospective case series, Annals of Internal Medicine, 171, 453-457, https://doi.org/ 10.7326/M19-0618.

Sebille E. van, Wilcox C., Lebreton L., Maximenko N., Hardesty B.D., Franeker J.A. van, Eriksen M., Siege D., Galgani F., Law K.L., 2015. A global inventory of small floating plastic debris, Environmental Research Letters, 10, 12, 124006, https://doi.org/10.1088/1748-9326/10/12/ 124006. 
Ter Halle A., Jeanneau L., Martignac M., Jardé É., Pedrono B., Brach L., Gigault J., 2017. Nanoplastic in the North Atlantic Subtropical Gyre, Environmental Science \& Technology, 51, 23, 13689-13697, https://doi.org/10.1021/acs. est. $7 \mathrm{~b} 03667$.

Thiel M., Luna-Jorquera G., Álvarez-Varas R., Gallardo C., Hinojosa I.A., Luna N., Miranda-Urbina D., Morales N., Ory N., Pacheco A.S., Portflitt-Toro M., Zavalaga C., 2018. Impacts of marine plastic pollution from continental coasts to subtropical gyres: fish, seabirds, and other vertebrates in the SE Pacific, Frontiers in Marine Science, 5, 238, https:// doi.org/10.3389/fmars.2018.00238.

Van der Wal M., van der Meulen M., Tweehuijsen G., Peterlin M., Palatinus A., Kovac Virsek M., Coscia L., Krzan A., 2015. Identification and assessment of riverine input of (marine) litter. Final report for the European Commission DG Environment, Framework contract No ENV.D.2/FRA/ 2012/0025, Bristol, Eunomia Research \& Consulting Ltd.
Veblen T., 1970 [1 $1^{\text {ere }}$ éd. 1901]. Théorie de la classe de loisir, Paris, Gallimard.

Weber M., 1965 [1 ${ }^{\text {ere }}$ éd. 1904]. L'objectivité de la connaissance dans les sciences et la politique sociales, in Essais sur la théorie de la science, Paris, Plon.

Weiss L., Ludwig W., Heussner S., Canals M., Ghiglione J.-F., Estournel C., Constant M., Kerhervé P., 2021. The missing ocean plastic sink: gone with the rivers, Science, 373, 6550, 107-111, https://doi.org/10.1126/science.abe0290.

Wilcox C., Sebille E. van, Hardesty B.D., 2015. Threat of plastic pollution to seabirds is global, pervasive, and increasing, Proceedings of the National Academy of Sciences, 112, 11899-11904, https://doi.org/10.1073/ pnas. 1502108112.

Zettler E.R., Mincer T.J., Amaral-Zettler L.A., 2013. Life in the "plastisphere": microbial communities on plastic marine debris, Environmental Science and Technology, 47, 13, 7137-7146, https://doi.org/10.1021/es401288x.

Citation de l'article: Blot D., Tramoy R., Gasperi J., Tassin B. Le continent oublié. Lumières et zones d'ombre des recherches sur la dissémination des plastiques. Nat. Sci. Soc., 29, 4, 469-478. 